\title{
Severe Thrombocytopenia in COVID-19: A Conundrum in Dengue-endemic Areas
}

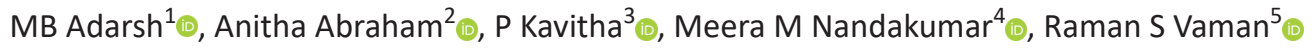

\begin{abstract}
Thrombocytopenia in coronavirus disease-2019 (COVID-19) can be attributed to multiple factors. Most often it is disease related. It is usually mild and if severe often associated with severe COVID-19 disease. It can also be due to drugs (Remdesivir, Tocilizumab) or coinfection with other viruses. Here we report two cases of severe thrombocytopenia in COVID-19 due to dengue coinfection. Most often the thrombocytopenia in dengue is self-resolving, and a careful "wait and watch" should suffice unlike COVID-19, where steroids can help if the cytopenia is due to cytokine storm or immune-mediated effects.

Keywords: COVID-19, Dengue, Thrombocytopenia.

Indian Journal of Critical Care Medicine (2021): 10.5005/jp-journals-10071-23778
\end{abstract}

\section{INTRODUCTION}

Thrombocytopenia in coronavirus disease-2019 (COVID-19) can be attributed to multiple factors. Most often it is disease related. It can be due to the reduced production of platelets from bone marrow because of cytokine storm or direct viral infiltration of the marrow and due to the increased peripheral destruction from immunemediated effects or excessive peripheral consumption as a result of microthrombi formation. ${ }^{1}$ Thrombocytopenia in COVID-19 is usually mild and if severe it is often associated with severe COVID19 disease. $^{2}$ The mean platelet count varied from $164 \times 10^{3} / \mathrm{mm}^{3}$ to $196 \times 10^{3} / \mathrm{mm}^{3}$ among various cohorts of COVID-19 cases. Severe life-threatening thrombocytopenia was not seen in these cohorts. There are many isolated case reports of severe thrombocytopenia in COVID-19 and were mostly attributed to immune-mediated peripheral destruction. ${ }^{3-5}$ It can also be due to drugs (Remdesivir, Tocilizumab) or coinfection with other viruses. Dengue is a mosquitoborne viral illness that is endemic in many countries and its incidence is worrisome in our area during this pandemic. ${ }^{6}$ Here we report two cases of severe thrombocytopenia in COVID-19 due to dengue coinfection. Most often the thrombocytopenia in dengue is selfresolving, and a careful "wait and watch" should suffice unlike COVID19 , where steroids can help if the cytopenia is due to cytokine storm or immune-mediated effects.

\section{Case Description}

Two patients presented with dengue coinfection. First was a 34-year male who presented with fever and headache for one day. His oropharyngeal swab for SARS-CoV-2 PCR was positive. His investigations showed a total leucocyte count of $2.9 \times 10^{9} / \mathrm{L}$ with $27 \%$ lymphocytes and a platelet count of $53 \times 10^{9} / \mathrm{L}$. The second case was a 54-year male with fever and severe body ache for 3 days. His oropharyngeal swab for SARS-CoV-2 PCR was positive. His total leucocyte count was $4.8 \times 10^{9} / \mathrm{L}$ with $40 \%$ lymphocytes and platelet count was $20 \times 10^{9} / \mathrm{L}$. There were no bleeding manifestations, and both were hemodynamically stable. A rapid ELISA for dengue NS1 antigen was positive in both cases. First case was managed symptomatically with paracetamol and oral fluids. His platelet count normalized in 5 days. The second case

\footnotetext{
${ }^{1}$ Department of General Medicine, Government Medical College, Kasaragod, Kerala, India

${ }^{2}$ Department of Community Medicine, Government Medical College, Kasaragod, Kerala, India

${ }^{3}$ Department of Anaesthesia, Government Medical College, Kasaragod, Kerala, India

${ }^{4}$ Department of ENT, Government Medical College, Kasaragod, Kerala, India

${ }^{5}$ Medical Superintendent, Government Medical College, Kasaragod, Kerala, India

Corresponding Author: MB Adarsh, Department of General Medicine, Government Medical College, Kasaragod, Kerala, India, e-mail: adarshmb@gmail.com

How to cite this article: Adarsh MB, Abraham A, Kavitha P, Nandakumar MM, Vaman RS. Severe Thrombocytopenia in COVID-19: A Conundrum in Dengue-endemic Areas. Indian J Crit Care Med 2021;25(4):465-466. Source of support: Nil

Conflict of interest: None
}

was given dexamethasone $6 \mathrm{mg}$ for 5 days, and his platelet count normalized in 6 days. Both had no other complications of dengue or COVID-19. The repeat nasopharyngeal swab for rapid antigen test SARS-CoV-2 was negative on the 10th day in both cases and were discharged.

Thrombocytopenia often alerts the physician in COVID19. Dengue coinfection can be a cause for it. This can cause severe thrombocytopenia and can be life-threatening. Early identification of coinfection can help the physician in anticipating other dengue-related complications like dengue shock syndrome and dengue hemorrhagic fever, which otherwise would have been attributed to severe COVID-19 and the treatment would have been initiated as for severe COVID19. Few things can point to non-COVID reasons for severe thrombocytopenia in COVID-19 cases. Fever, headache, body ache, and severe fatigue are common to both the viral illnesses. Unlike dengue, skin manifestations are rare in COVID-19. 
Dengue fever is characterized by maculopapular and diffuse erythematous rash in more than $50 \%$ of patients. ${ }^{8}$ Presence of rash with thrombocytopenia in COVID-19 should alert a physician in this regard. Also, disease-related thrombocytopenia in COVID-19 is often associated with severe disease. If the patient is asymptomatic or mildly symptomatic for COVID-19, other causes like coinfection with dengue as in the index cases should be thought of for severe thrombocytopenia. In both the index, patients were only mildly symptomatic for COVID19. Despite the dengue coinfection, COVID-19 did not worsen and there was no persistence of antigen positivity. This may be due to the enhanced nonspecific antiviral response elicited by the coinfection. This possible synergistic effect needs to be studied further. But this is not always the case. Few deaths have been reported due to the coinfection. ${ }^{9}$ Making the diagnosis of coinfection is also difficult as false-positive serological tests for dengue have been seen in COVID-19 patients. ${ }^{10}$ So, we will have to go with antigen-based tests for a proper diagnosis.

In conclusion, dengue coinfection can cause severe thrombocytopenia in COVID-19 and identifying it early can help in the better management of patients.

\section{Highlights}

- Dengue coinfection can cause severe thrombocytopenia in COVID-19.

- Dengue-COVID-19 coinfection and severe thrombocytopenia had good outcome at discharge.

\section{ORCID}

MB Adarsh (1) https://orcid.org/0000-0001-8091-1952

Anitha Abraham (1) 0000-0002-1386-4241

Kavitha (1) 0000-0001-6288-5906

Meera M Nandakumar (1) 0000-0003-4733-9135

Raman Swathy Vaman (10000-0003-0709-4110

\section{References}

1. Xu P, Zhou Q, Xu J. Mechanism of thrombocytopenia in COVID-19 patients. Ann Hematol 2020;99(6):1205-1258. DOI: 10.1007/s00277020-04019-0.

2. Lippi G, Plebani M, Henry BM. Thrombocytopenia is associated with severe coronavirus disease 2019 (COVID-19) infections: a meta-analysis. Clin Chim Acta 2020;506:145-148. DOI: 10.1016/j. cca.2020.03.022.

3. Sadr S, SeyedAlinaghi S, Ghiasvand F, Hassan Nezhad M, Javadian N, Hossienzade R, et al. Isolated severe thrombocytopenia in a patient with COVID-19: a case report. IDCases 2020;21:e00820. DOI: 10.1016/j. idcr.2020.e00820.

4. Chen W, Yang B, Li Z, Wang P, Chen Y, Zhou H. Sudden severe thrombocytopenia in a patient in the recovery stage of COVID-19. Lancet Haematol 2020;7(8):e624. DOI: 10.1016/S2352-3026(20)30175-7.

5. Malik J, Javaid M, Majedi O, Ishaq U, Zahid T. Paying in blood: a case of thrombocytopenia in Covid-19. Cureus 2020;12(8):e9791. DOI: 10.7759/cureus.9791.

6. Varghese B, Kumaran JA. Trend of dengue fever in COVID-19 period: observation from Kerala, India. Int J Commun Med Publ Health 2020;7(8):3307. DOI: 10.18203/2394-6040.ijcmph20203419.

7. Wollina U, Karadağ AS, Rowland-Payne C, Chiriac A, Lotti T. Cutaneous signs in COVID -19 patients: a review. Dermatol Ther 2020;33(5):e13549. DOI: 10.1111/dth.13549.

8. Thomas E, John M, Kanish B. Mucocutaneous manifestations of dengue fever. Indian J Dermatol 2010;55(1):79. DOI: 10.4103/00195154.60359.

9. Miah MDA, Husna A. Coinfection, coepidemics of COVID-19, and dengue in dengue-endemic countries: a serious health concern. J Med Virol 2020. Online ahead of print. DOI: 10.1002/jmv.26269.

10. Yan G, Lee CK, Lam LTM, Yan B, Chua YX, Lim AYN, etal. Covert COVID-19 and false-positive dengue serology in Singapore. Lancet Infect Dis 2020;20(5):536. DOI: 10.1016/S1473-3099(20)30158-4. 